\title{
Primary Extra-Gastrointestinal Stromal Tumor (GIST) arising from mesentery of small bowel and presenting as abdominal mass: A rare entity
}

\author{
Alok Kumar Tiwari ${ }^{*}$, Anil Kumar Choudhary ${ }^{2}$, Hemant Khowal ${ }^{1}$, Poras Chaudhary ${ }^{1}$, \\ Mohinder P. Arora \\ ${ }^{1}$ Department of Surgery, Lady Hardinge Medical College, New Delhi, India \\ ${ }^{2}$ Department of Surgery, Dr R.M.L Hospital, New Delhi, India \\ Email: dralokty1@gmail.com, dranil@gmail.com, hemantkh12@gmail.com, poraschaudhary1@gmail.com, mparora22@gmail.com
}

Received 20 July 2013; revised 21 August 2013; accepted 29 August 2013

Copyright (C) 2013 Alok Kumar Tiwari et al. This is an open access article distributed under the Creative Commons Attribution License, which permits unrestricted use, distribution, and reproduction in any medium, provided the original work is properly cited.

\begin{abstract}
Introduction: Majority of mesenchymal tumors of gastrointestinal tract are Gastrointestinal Stromal Tumor (GIST). It is, however, a rare tumor, accounting for less than $1 \%$ of primary gastrointestinal (GI) neoplasms. Though, these tumors are refractory to conventional chemotherapy or radiotherapy but show a good response to targeted adjuvant chemotherapy with tyrosine kinase inhibitors following surgical resection. Case Report: we report here a case of primary Extra-GIST tumor arising from mesentry of small bowel near duodeno-jejunal junction in a 69 years old male patient. The patient presented with a palpable mass in upper abdomen for past 15 days. On examination, a non-tender mobile lump of size around $17 \times 10 \mathrm{~cm}$, with bosselated surface and firm in consistency was palpable involving epigastric, left hypochondrium and umbilical region. Contrast enhanced computed tomography of abdomen revealed a heterogenous mesentric mass. On surgical intervention a mass was found involving mesentery near dudenojejunal junction without involvement of gastrointestinal tract. Complete surgical resection of the tumor was done and adjuvant chemotherapy with Imatinib mesylate was started as HPE revealing GIST with mitotic index of $>10 / 50 \mathrm{HPF}$ and $17 \times 10 \mathrm{~cm}$ size placed the patient in high risk category. Patient was discharged on 12th of post-operative day with advice of regular follow-up. Conclusion: GIST occurrence is not restricted to bowel but can involve unusual sites also. The mainstay of treatment remains surgical resection with adequate margin. In cases where tumour has malignant potential (high mitotic figures on histo-
\end{abstract}

"Corresponding author. pathology) adjuvent treatment with tyrosine kinase may prevent or delay relapse.

Keywords: Extra-Gastrointestinal Stromal Tumors; GIST; Mesentric Tumors; Imatinib Mesylate; Abdominal Mass

\section{INTRODUCTION}

Gastrointestinal Stromal Tumors coined by Mazur and Clark are the most common mesenchymal tumors of gastrointestinal tract found to be immunohistochemically and ultrastructurally different from other spindle cell tumors [1]. GISTs can occur anywhere in the gastrointestinal (GI) tract, commonest site being stomach (Approximately $60 \%-70 \%$ of GISTs), followed by small intestine $(25 \%-35 \%)$, colon, rectum, appendix (together $5 \%)$, and esophagus $(2 \%-3 \%)$. Rarely, they may arise from the mesentery or omentum [2].

The clinical manifestations of GISTs depend on the location and size of the tumors and are often nonspecific although patients with advanced disease may present with symptoms of a mass lesion, abdominal pain, or bleeding $[3,4]$.

The availability of the KIT tyrosine kinase inhibitor (STI-571, imatinib/Gleevec/Novartis) has revolutionized the treatment of gastrointestinal stromal tumors, thereby making it important to know this disease entity.

We report here a case of extra-gastrointestinal stromal tumor arising from mesentery of small intestine presenting as an abdominal mass.

\section{CASE REPORT}

A 69 years old male patient presented with history of 
progressively increasing mass in upper abdomen without any other significant complaints for last 15 days. When evaluated clinically patient was found haemodynamically stable and his per-abdominal examination revealed a mass of size $17 \times 10 \mathrm{~cm}$ which was mobile, non-tender, with bosselated surface and firm in consistency involving epigastric, left hypochondrium and umbilical region.

His laboratory workups were unremarkable and Contrast Enhanced Computed Tomography (CECT) of abdomen depicted a heterogenous mesentric mass (Figure 1).

On laparotomy, $17 \times 10 \mathrm{~cm}$ mass involving the mesentery (Figure 2) near the duodeno-jejunal junction was found without any gross evidence of gastrointestinal tract involvement, peritoneal deposits, ascitis and lymph node enlargement. Resection of the tumor along with the mesentery and ligation of the feeding vessels was carried out and specimen was sent for histopathological examination.

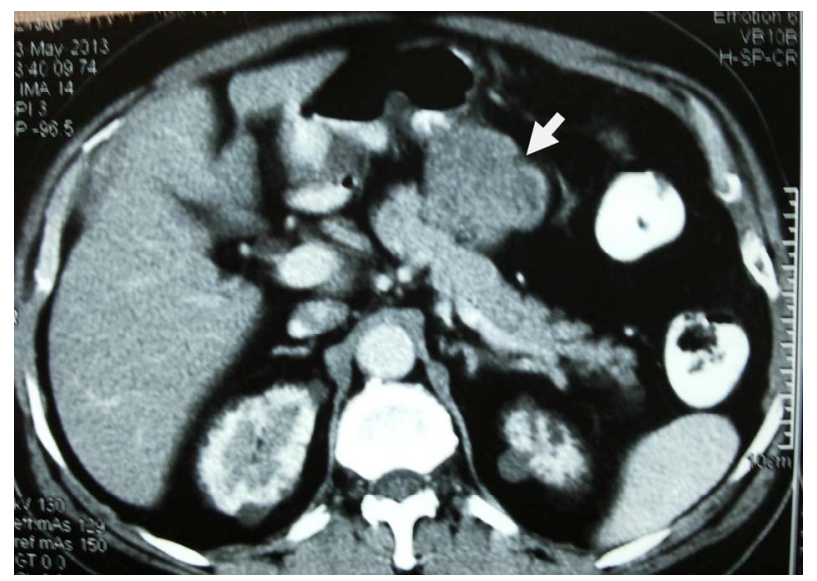

Figure 1. CECT abdomen showing a heterogenous mass (arrow).

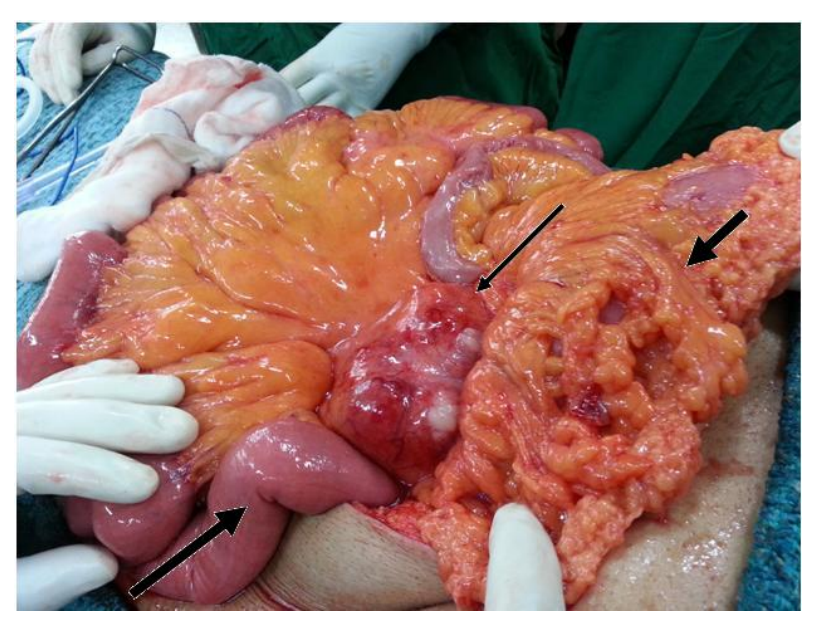

Figure 2. Large mesenteric mass near duodeno-jejunal junction (thin arrow) away from transverse colon (thick short arrow) and small intestine (thick long arrow).
Histopathological examination grossly revealed grey white soft tissue mass of size $17 \times 10 \mathrm{~cm}$ with encapsulated and nodular external surface. Microscopical examination showed features of malignant spindle cell tumors arranged in short fascicles and focally forming whorls with myxoid areas and multiple foci of necrosis. Individual tumor cells revealed moderate nuclear pleomorphism, brisk mitosis ( $>10 / \mathrm{HPF})$. On the basis of immunohistochemistry, the tumor cells were found to be positive for Vimentin/smooth muscle actin/(SMA) and CD-117.

The patient was discharged on 12th of post operative day on Imatinib mesylate adjuvent chemotherapy and is on regular follow up.

\section{DISCUSSION}

Gastro-Intestinal Stromal Tumor (GIST) appears to arise from the interstitial cell of Cajal $[5,6]$ but the exact cell of origin and precise steps in tumorogenesis are not well established. However, mutation in the proto-oncogene $c$-kit leading to increased expression of KIT (type III tyrosine kinase receptor) and platelet-derived growth factor receptor-alpha (PDGFRA, found in 5\% - 10\%) and loss of heterozygosity of the NF1 gene are thought to play a major role.

Microscopically GISTs are classified into: spindle cell type $(70 \%)$, epitheloid type $(20 \%)$, and mixed spindle cell and epithelioid cell type. On immunohistochemical staining, 95\% are CD117 (c-kit) positive, 70\% are CD34, and $40 \%$ stain positive for smooth muscle actin.

Liver and peritoneum are the most common sites of metastasis via hematogenous route while metastasis to the lung, bones, and lymph nodes is rare.

GISTs may grow into large size by displacing adjacent structures rather than invading them and so they are symptomatic where as small tumors are asymptomatic. The symptoms expressed are non-specific GI symptoms such as nausea, vomiting, dyspepsia, abdominal pain, distension, or change in bowel behavior. The symptoms of obstruction, bleeding, or rupture into the peritoneal cavity are less common.

GISTs initially presenting as an abdominal mass are exceedingly rare, and only 21 such cases including 4 cases involving only mesentery as primary site have been reported in the world literature since 2001 [7-10].

In accordance with all larger clinicopathologic series, GISTs have a predilection to adults over 50 years of age with the median ages varying between 55 - 65 years in different sites with no clear sex predilection. Whereas the proportion of patients under 40 years of age ranged between $5 \%$ and $20 \%$ but they are reported to be extremely rare in children.

A number of GISTs have been reported outside the 
Gastrointestinal (GI) tract in the abdomen as a result of metastasis from GI-tract proper specifically in omentum, mesenteries, retroperitoneum and urinary bladder serosa $[11,12]$. However, GIST originating from these sites as a primary tumor is rare. The primary site of disseminated intra-abdominal GIST involving multiple intestines, peritoneal surfaces and other abdominal organ is often impossible to ascertain. More commonly, GISTs in these locations represents intra-abdominal metastases from gastric or intestinal primaries. Search of origin of primary tumor whether it is from stomach or intestines is always necessary for apparent extra-GI GISTs.

Small GISTs are often firm and sometimes rubbery, whereas larger and malignant GISTs tend to be soft with fish-flesh or lymphoma-like consistency.

In order to carry out detection, staging, surgical planning and follow-up of patients with GIST, CT, MRI and fluorine-18-fluorodeoxyglucose (FDG) positron emission tomography (PET) are considered to be the imaging modality of choice. The majority of GISTs appear to be welldefined, extraluminal or intramural masses with varying attenuation on CT. Small lesions, which are usually benign, tend to be well-defined and relatively homogeneous, While larger lesions normally show well-defined or ill-defined margins, inhomogeneous density both on unenhanced and on contrast-enhanced scans and a tendency to spread to surrounding structures. Large tumors $(>6 \mathrm{~cm})$ frequently show central areas of necrosis or haemorrhage [13].

Primary gastrointestinal stromal tumor in the omentum and mesentery can be suggested as a diagnosis in a patient with a well-marginated, lobulated mass that contains large areas of low attenuation and lacks central gas. The imaging appearance of mesenteric and omental GISTs is indistinguishable from that of other sarcomas that may arise in these locations [11].

Unlike gastrointestinal adenocarcinomas, GISTs metastasizing to the lymph nodes are extremely rare. The CT characteristics of metastatic lesions of GISTs are similar to those of primary tumors, enhancing masses that can be heterogeneous because of necrosis, hemorrhage, or cystic degeneration.

\section{TREATMENT}

The treatment goal for localized primary GIST is complete resection followed by adjuvant chemotherapy without the need for lymphadenectomy or wide resection margins.

Neoadjuvant Imatinib is not recommended where a change in tumor size will not affect surgery [14]. It can, however, be considered where a tumor response could permit function-sparing surgery, e.g., rectum or oesophagus.
The tumor should be removed en bloc with a clear margin. The pseudocapsule should be removed and not be penetrated. Therefore, either a wedge resection in case of stomach or segmental resection in case of intestine is required.

Lymph node dissection or biopsy is not recommended mainly due to the pattern of spread of GISTs because lymph node metastases are rare.

GIST shows a very dramatic response to a Tyrosine kinase inhibitor, Imatinib mesylate (STI-571/Gleevec/ Novartis/Basel). This drug is a molecular targeted therapy, and acts on the c-kit growth factor receptor, which is the most important diagnostic marker of GISTs. This drug also inhibits several other tyrosine kinase receptors with varying affinity as also the BCR-ABL fusion protein and the platelet derived growth factor receptor (PDGFR). The cases resistant to Imatinib or showing progression can be controlled by sunitinib malate (SU11248/ Sutent).

In general practice, contrast-enhanced CT is routinely used to monitor tumor response following treatment with tyrosine kinase inhibitors. The degree and pattern of enhancement observed on CT scans are useful for identifying post treatment changes. On Contrast-Enhanced Computed Tomography (CECT), a response to Imatinib is characterized by rapid transition from a heterogeneously hyper-attenuating pattern to a homogeneously hypo-attenuating pattern with resolution of the enhanced tumor nodules and a decrease in tumor vessels.

The density of hepatic metastases decreases after treatment to approximately $20-25 \mathrm{H}$, which is near to, but greater than that for a true cyst $(<15 \mathrm{H})$. Follow-up CT scans showing that the mass has become homogeneous with significant decreases in the attenuation and size of the tumor indicate a good response to imatinib [13].

Tumor size, anatomic location, and mitotic count are considered independent prognostic factors for GISTs $[15,16]$ and tumors that measure $\geq 5 \mathrm{~cm}$ in size are associated with an unfavorable prognosis. The anatomic site of origin of a GIST is associated strongly with its clinical behavior. The patients who have tumors arising in the rectum or small intestine have the worst prognosis as compared to the patients who have esophageal and gastric neoplasms [16]. Likewise, GISTs that exhibit $\geq 5$ mitoses per $50 \mathrm{HPF}$ or $\geq 2$ mitotic figures per $10 \mathrm{HPF}$ are associated with an unfavorable prognosis regardless of their site of origin $[15,17]$.

In a series of 200 GISTs, median survival was 66 months for complete resection compared with 22 months for incomplete resection or unresectable disease [18].

Spontaneous tumor rupture, or rupture during surgery, increases the risk of peritoneal recurrence and is an adverse prognostic factor. Overall 5-year survival rates for primary resected disease are in the order of $50 \%-55 \%$. 
Most recurrences occurs within the first 2 years of resection necessitating the regular follow up of these patients.

A high risk patient should have a CT scan every 3 - 4 months for 3 years, then every 6 months to 5 years. For low risk, a CT scan every 6 months for 5 years is acceptable [14].

GISTs of the jejunum and ileum treated surgically have been shown to have a $39 \%$ tumor related mortality, which was twice that of gastric GISTs [19].

Our case had mesenteric stromal tumor near duodenojejunal junction, mitoses were $>10$ per $50 \mathrm{HPF}$ and total tumor diameter was $17 \mathrm{~cm}$. The localization was unfavorable but bad prognostic signs such as liver or lymph node metastases were not seen.

Gene expression patterns in GISTs are assessed by DNA microarray techniques. The technique revealed that the gene FLJ10261 responsible for encoding the DOG1 protein is specifically expressed in GISTs, irrespective of KIT or PDGFRA mutation status. However, its function is not well understood, although it seems to be fairly specific to GIST and rarely being expressed in other soft tissue tumors. In future it may play a pivotal role in diagnosis of GISTs, especially in PDGFR mutants failing to express the KIT antigen [20].

\section{CONCLUSION}

GIST occurrence is not restricted to bowel but can involve unusual sites also and involvement of mesentery near dudeno-jejunal junction is very rare. The mainstay of treatment remains surgical resection with adequate margin. In cases where tumor has malignant potential based on high mitotic figures on histopathology adjuvent treatment with tyrosine kinase may prevent or delay relapse. DNA microarray technique may play a vital role in identifying the gene encoding DOG1 protein in mutants that does not express KIT antigen and thus may help in its diagnosis.

\section{AUTHOR'S CONTRIBUTIONS}

All authors have contributed to patient management, writing the case report, reviewing and have given the final approval for publishing the manuscript.

\section{REFERENCES}

[1] Mazur, M.T. and Clark, H.B. (1983) Gastric stromal tumours. Reappraisal of their histogenesis. American Journal of Surgical Pathology, 7, 507-519. doi:10.1097/00000478-198309000-00001

[2] Miettinen, M. and Lasota, J. (2001) Gastrointestinal stromal tumors: Definition, clinical, histological, immunohistochemical, and molecular genetic features and differential diagnosis. Virchows Arch, 438, 1-12.

\section{doi:10.1007/s004280000338}

[3] Filippou, D.K., Pashalidis, N., Skandalakis, P. and Rizos, S. (2006) Malignant gastrointestinal stromal tumor of the ampulla of Vater presenting with obstructive jaundice. Journal of Postgraduate Medicine, 52, 204-206.

[4] Towu, E. and Stanton, M. (2006) Gastrointestinal stromal tumour presenting with severe bleeding: A review of the molecular biology. Pediatric Surgery International, 22, 462-464. doi:10.1007/s00383-006-1636-5

[5] Torihashi, S., Nishi, K., Tokutomi, Y., Nishi, T., Ward, S. and Sanders, K.M. (1999) Blockade of kit signaling induces transdifferentiation of interstitial cells of Cajal to a smooth muscle phenotype. Gastroenterology, 117, 140148. doi:10.1016/S0016-5085(99)70560-3

[6] Young, H.M., Ciampoli, D. and Southwell Newgreen, D.F. (1996) Origin of interstitial cells of Cajal in the mouse intestine. Developmental Biology, 180, 97-107. doi:10.1006/dbio.1996.0287

[7] Sinha, R., Verma, R. and Kong, A. (2004) Mesenteric gastrointestinal stromal tumor in a patient with neurofibromatosis. American Journal of Roentgenology, 183, 1844-1846. doi:10.2214/ajr.183.6.01831844

[8] Basile, A., Kettenbach, J., Mundo, E., et al. (2006) Erratum: Primitive mesenteric gastrointestinal stromal tumor with autonomic nerve/ganglionic differentiation presenting as a huge mass with small synchronous nodules. European Radiology, 16, 519. doi:10.1007/s00330-005-2845-3

[9] Gupta, N., Mittal, S., Lal, N., Misra, R., Kumar, L. and Bhalla, S. (2007) A rare case of primary mesenteric gastrointestinal stromal tumor with metastasis to the cervix uteri. World Journal of Surgical Oncology, 5, 137. doi:10.1186/1477-7819-5-137

[10] Patil, S., Jain, S., Kaza, R.C.M. and Chamberlain, R.S. (2011) Giant gastrointestinal stromal tumor presenting as a palpable abdominal mass: An unusual presentation. ISRN Surgery, 2011, Article ID: 894829.

[11] Miettinen, M., Monihan, J.M., Sarlomo-Rikala, M., Kovatich, A.J., Carr, N.J., Emory, T.S. and Sobin, L.H. (1999) Gastrointestinal stromal tumors/smooth muscle tumors/GISTs in the omentum and mesentery-Clinicopathologic and immunohistochemical study of 26 cases. American Journal of Surgical Pathology, 23, 1109-1118. doi:10.1097/00000478-199909000-00015

[12] Reith, J.D., Goldblum, J.R., Lyles, R.H. and Weiss, S.W. (2000) Extragastrointestinal (soft tissue) stromal tumors. An analysis of 48 cases with GIST 23 emphasis on histological predictors of outcome. Modern Pathology, 13, 577-585. doi:10.1038/modpathol.3880099

[13] Chourmouzi, D., Sinakos, E., Papalavrentios, L., Akriviadis, E. and Drevelegas A. (2009) Gastrointestinal stromal tumors: A pictorial review. Journal of Gastrointestinal and Liver Diseases, 18, 379-383.

[14] Blay, J.Y., Bonvalot, S., Casali, P., et al. (2005) Consensus meeting for the management of gastrointestinal stromal tumors. Annals of Oncology, 16, 993.

[15] Cunningham, R.E., Federspiel, B.H., McCarthy, W.F., Sobin, L.H. and O'Leary, T.J. (1993) Predicting progno- 
sis of gastrointestinal smooth muscle tumors. Role of clinical and histologic evaluation, flow cytometry, and image cytometry. American Journal of Surgical Pathology, 17, 588-594.

doi:10.1097/00000478-199306000-00006

[16] Emory, T.S., Sobin, L.H., Lukes, L., Lee, D.H. and O'Leary, T.J. (1999) Prognosis of gastrointestinal smooth-muscle (stromal) tumors. Dependence on anatomic site. American Journal of Surgical Pathology, 23, 82-97. doi:10.1097/00000478-199901000-00009

[17] Miettinen, M., Sarlomo-Rikala, M., Sobin, L.H. and Lasota, J. (2000) Esophageal stromal tumors: A clinicopathologic, immunohistochemical, and molecular genetic study of 17 cases and comparison with esophageal leiomyomas and leiomyosarcomas. American Journal of Surgical Pathology, 24, 211-222. doi:10.1097/00000478-200002000-00007
[18] DeMatteo, R.P., Lewis, J.J., Leung, D., et al. (2000) Two hundred gastrointestinal stromal tumors: Recurrence patterns and prognostic factors for survival. Annals of Surgery, 231, 51-58. doi:10.1097/00000658-200001000-00008

[19] Miettinen, M., Makhlouf, H., Sobin, L.H., et al. (2006) Gastrointestinal stromal tumors of the jejunum and ileum: A clinicopathologic, immunohistochemical, and molecular genetic study of 906 cases before imatinib with longterm follow-up. American Journal of Surgical Pathology, 30, 477-489. doi:10.1097/00000478-200604000-00008

[20] Allander, S.V., Nupponen, N.N., Rigner, M., Hostetter, G., Maher, G.W., Goldberger, N., Chen, Y., Carpten, J., Elkahloun, A.G. and Meltzer, P.S. (2001) Gastrointestinal stromal tumors with KIT mutation exhibit a remarkably homogenous gene expression profile. Cancer Research, 61, 8264-8268. 